\title{
Reflexiones en torno a la ética, la inteligencia humana y la inteligencia artificial
}

José L. García-Vigil*

Universidad Nacional Autónoma de México, Facultad de Medicina, Departamento de Farmacología, Ciudad de México, México

\section{Resumen}

En internet ha crecido la inteligencia artificial hasta convertirse en un programa con códigos y algoritmos que aprenden y se reprograman para efectuar tareas preestablecidas con mayor eficiencia; si bien lo anterior se traduce en mejoría, el programador desconoce los alcances de los resultados y de la reprogramación. Ante el riesgo de desviación de los objetivos preestablecidos y de los reglamentos éticos, se tienen que implementar filtros al inicio, durante y al final del proceso, como alarmas cuando existan desviaciones con implicación bioética. La interacción de la inteligencia humana con la inteligencia artificial ha tenido desencuentros negativos y positivos. Al principio, bastó con adecuar normas, leyes laborales y derechos humanos; ahora se requiere establecer normas éticas, como las formuladas en la Declaración de Barcelona para el Adecuado Desarrollo y Uso de la Inteligencia Artificial en Europa.

PALABRAS CLAVE: Inteligencia artificial. Inteligencia humana. Normas éticas. Edición bioética.

\section{Reflections around ethics, human intelligence and artificial intelligence}

\begin{abstract}
On the internet, artificial intelligence has grown to become a program with codes and algorithms that learn and reprogram themselves to carry out pre-established tasks with greater efficiency; although this translates into improvements, the scope of the results and reprogramming are unknown to the programmer. Given the risk of deviation from pre-established objectives and ethical regulations, filters must be installed at the beginning, during and at the end of the process, as alarms for detecting deviations with bioethical implications. The interaction of human intelligence with artificial intelligence has had negative and positive disagreements. Initially, adapting regulations, labor laws and human rights was enough; now it is necessary for ethical standards to be established, such as those formulated in the Barcelona Declaration for the Proper Development and Usage of Artificial Intelligence in Europe.
\end{abstract}

KEY WORDS: Artificial intelligence. Human intelligence. Ethical standards. Bioethics edition.

\section{Antecedentes evolución histórica de la IA e implicaciones éticas iniciales}

La denominada inteligencia artificial (IA) tiene una larga historia, la cual parte de la década de 1960 cuando la informática tuvo un desarrollo mayor al pasar del procesamiento semiautomático de la información con el uso de calculadoras para usos comerciales y científicos, a la plena automatización realizada por medio de equipos (hardware) y sistemas operativos (software) que permitieron una mecanización más rápida y exacta de la información, al principio
Correspondencia:

*José L. García-Vigil

E-mail: pataloca5005@gmail.com
Fecha de recepción: 10-11-2020

Fecha de aceptación: 13-04-2021

DOI: $10.24875 / G M M .20000818$
Gac Med Mex. 2021;157:311-314

Disponible en PubMed

www.gacetamedicademexico.com

0016-3813/@ 2021 Academia Nacional de Medicina de México, A.C. Publicado por Permanyer. Este es un artículo open access bajo la licencia CC BY-NC-ND (http://creativecommons.org/licenses/by-nc-nd/4.0/). 
especialmente en cálculos matemáticos y estadísticas $\mathrm{y}$, posteriormente, en procesamiento de palabras y textos más elaborados.

Hasta ese momento del desarrollo de la informática computacional, no había mayor problema normativo ni de índole ético para su aceptación, pues apoyaba al humano en muchas tareas administrativas y matemáticas rutinarias y repetitivas, las cuales se podían realizar con mayor rapidez, liberando así a los individuos para que orientaran más tiempo a la realización de tareas intelectuales y científicas. Este momento es el punto de inflexión cuando la mente se liberó y el ser humano decidió expandir su intelecto con base en la naciente IA.'

De este punto de inflexión con expansión de la mente humana y subordinación de tareas rutinarias al ordenador o computadora individual de escritorio, lo único por reglamentar era el tiempo y tipo de trabajo por desarrollar con dicho equipo, previa capacitación para, por ejemplo, la elaboración y reproducción de documentos en formatos electrónico (cartas, mensajes, memorandos, informes directivos y de supervisión, trabajos científicos, financieros y mercantiles o comerciales, etc.), así como para su distribución y resguardo en bases de datos y archivos; asimismo, se requirió capacitación en el uso de telecomunicaciones automatizadas e internet (correo electrónico, llamadas telefónicas, mensajería en general).

¿Qué era necesario hacer en ese momento? Capacitar al personal en las empresas y formular la reglamentación administrativa para definir puestos de trabajo a fin de proteger la información y resguardar derechos laborales con mediación entre directivos y representantes sindicales. En este paso del desarrollo de la IA, la implicación ética estaba relacionada con los derechos laborales y los derechos humanos de los trabajadores.

\section{Desarrollo contemporáneo de la IA y nuevos conflictos éticos}

El siguiente desarrollo de la IA fue paralelo al del naciente internet, el cual pasó de usos científicos y militares limitados, al uso comercial, científico, laboral, educativo y cultural, en todo tipo de quehacer humano. Poco a poco, la subordinación de tareas repetitivas y rutinarias de la inteligencia humana se trasladó a la $\mathrm{IA}$, volviéndose esta prácticamente una prótesis de la primera. En este momento no había mayor conflicto ético en la coexistencia de la inteligencia humana y la artificial; su relación era amigable y, hasta cierto punto, más productiva en todo sentido. ${ }^{2,3}$
La innovación tecnológica y el mayor desarrollo de la IA fue derivando hasta automatizar prácticamente todas las tareas humanas en el manejo y procesamiento de la información, con lo que surgieron las llamadas tecnologías de la información y la comunicación (TIC), así como ciencias, técnicas y profesiones relacionadas con su aprendizaje, desarrollo, aplicación y realimentación en mayor innovación tecnológica con retroalimentación positiva. Con ello se hizo más aparente la relación ontológica de la ciencia con la tecnología. ${ }^{2,3}$

Esta relación ontológica virtuosa se fortaleció de tal manera que la ciencia al generar nuevos conocimientos, estos ya validados pasaban a formar parte del acervo cultural, científico e intelectual del humano. Asimismo, al aplicar la IA en el quehacer cotidiano con la automatización y fabricación de objetos electrónicos de uso doméstico (aparatos como televisión, radio, reproductor de música, teléfonos fijos, utensilios de cocina, computadoras de escritorio, teléfonos celulares, cámaras de vigilancia, reloj despertador, etcétera), la vida de las personas se hizo más cómoda.

Todavía no había mayor conflicto en la convivencia inteligencia humana con la IA, pero cuando se decidió que muchos de los puestos de trabajo fueran sustituidos por computadoras y no por nuevas personas ya capacitadas, la situación se tornó incómoda y la implicación ética, de mayor impacto. El efecto fue desempleo, requerimiento de mayor capacitación o formación en nuevas profesiones como en el uso de las TIC y ciencias de la computación e información automatizada. Este cambio se hizo patente a fines del siglo pasado y principios del presente.

El cambio máximo que decidió un ajuste en las reglas y normas laborales, además de la legislación financiera, comercial, mercantil, cultural y laboral, fue la sustitución del trabajo manual de personas por robots y computadoras en las telecomunicaciones para todo tipo de trámites administrativos y burocráticos, los cuales forman parte de los procesos de información que se verifican en instituciones existentes en una sociedad bien organizada: centrales de llamadas telefónicas por grabaciones que contestan y dan respuesta al tipo de requerimiento de información, o traslación de las llamadas a un menú de elecciones según la información solicitada, sin participación de persona alguna.

\section{Desarrollo contemporáneo de la IA y nuevas implicaciones éticas}

El golpe mayor se produjo cuando los expertos en códigos y algoritmos para la programación de IA 
decidieron ampliar sus horizontes a internet y a todos los dispositivos que no solo requirieran la automatización del trabajo manual y algo del intelectual. Ahora tratan de sustituir, o incluso superar, la creatividad humana por la artificial. Esta tendencia tiene la metáfora de la neurona como equivalente de una computadora inteligente y el cerebro, de redes inteligentes de computadora, operadas con programas de redes neuronales multinivel, analógicas y digitales, con códigos y algoritmos que permiten profundizar en el aprendizaje; se ha recodificado y reorientado la programación del software de IA a solución de problemas complejos, sin la intervención de la mente humana con su ya conocida y ponderada inteligencia, consciencia y creatividad. ${ }^{2,3}$

Si bien antes no había mucho problema ético ni normativo laboral por violación de derechos humanos o de trabajo, de 10 años a la fecha los nuevos desarrollos de la IA de aprendizaje profundo han llegado al límite de trastornar todo tipo de legislación del saber y quehacer humano; han puesto en duda la vigencia y validez de virtudes y valores, tanto éticos como morales, de la sana convivencia de la sociedad. ${ }^{4}$

Estos nuevos cambios implican no solo aprender y normar leyes y reglamentos para aprender y aplicar en forma adecuada la IA para beneficio de la inteligencia humana y como apoyo en la tarea intelectual y creativa del humano, sino que también se tiene que legislar y normar la formación y capacitación de los profesionales de las ciencias de la información automatizada, para que en la elaboración de nuevos programas de IA se consideren códigos deontológicos. ${ }^{5-9}$

Por otro lado, la aplicación de la IA en las telecomunicaciones, en especial en las llamadas redes sociales (Facebook, Instagram, Twitter, WhatsApp, entre varias más), ha incorporado un riesgo mayor para la sociedad, pues en forma clara y flagrante se viola la privacidad de las personas, a quienes se les da la falsa seguridad de relación y conexión, además de seguridad y simpatía de gran cantidad de "amigos", cuando en realidad se está creando aislamiento y adicción y se manipulan los sentimientos; los usuarios no son los clientes sino los producto que están en venta. $^{10}$

Asimismo, por muy inteligentes que sean las futuras IA, siempre serán distintas a la inteligencia humana que las ha creado. Además, el hecho de ser ajenas a los valores y necesidades humanas nos debería hacer reflexionar sobre aspectos éticos en su desarrollo y, en particular, sobre la conveniencia de dotar de total autonomía a las máquinas. ${ }^{11}$

\section{Normativa ética específica para desarrollo y aplicación de la IA en Europa}

Estos aspectos científicos y éticos justificaron la realización de una reunión para debatir el tema el 8 de marzo de 2017 en Barcelona, en la que participaron distintos expertos europeos en inteligencia artificial, computación y comunicación, entre otros ámbitos. El debate dio lugar a la Declaración de Barcelona para un Adecuado Desarrollo y Uso de la IA en Europa, la cual contiene básicamente los siguientes principios y valores: ${ }^{12}$

- Prudencia. El salto adelante en la IA ha sido causado por la maduración de las tecnologías de $I A$, la potencia informática y el almacenamiento de datos enormemente aumentados, la disponibilidad de plataformas de entrega a través de internet y una mayor disposición de muchos actores económicos para probar la tecnología para su propio dominio de aplicación.

- Fiabilidad. Todos los sistemas artificiales que se utilizan en nuestra sociedad deben someterse a pruebas para determinar su fiabilidad $y$ seguridad.

- Explicabilidad. Cuando un sistema de IA toma una decisión, los humanos afectados deben obtener una explicación de la razón de esa decisión en términos que puedan entender y deben poder desafiar la decisión con argumentos razonados.

- Responsabilidad. Existe una creciente preocupación por los chatbots de IA y otros tipos de sistemas de mensajería automática que operan en internet y en las redes sociales, diseñados para la manipulación de la opinión política, la desinformación mediante la propagación de hechos falsos, la extorsión u otras formas de actividad maliciosa que es peligrosa para las personas y desestabiliza a la sociedad.

- Autonomía restringida. Los sistemas de IA no solo tienen la capacidad de tomar decisiones. Cuando están integrados en sistemas físicos, como los automóviles autónomos, tienen el potencial de actuar sobre sus decisiones en el mundo real. Esto plantea preguntas sobre la seguridad y sobre si la IA autónoma no superará en algún momento a la inteligencia humana.

- Papel humano. El innegable entusiasmo actual por la IA a veces da la impresión de que la 
inteligencia humana ya no es necesaria y ha llevado a algunas organizaciones a despedir empleados y reemplazarlos por sistemas de IA, error grave ya que todos los sistemas de IA dependen críticamente de la inteligencia humana.

Finalmente, todas estas son llamadas de atención para que los mismos programas contengan códigos y algoritmos preventivos para detenerse o mandar señales de alerta ante desviaciones graves en el análisis y procesamiento de la información, las cuales pudieran provocar riesgos potenciales para la supervivencia sana y armónica de los humanos, incluso para que desaparezca totalmente la especie homo sapiens sapiens de la faz de la tierra. ${ }^{13}$

\section{Conflicto de intereses}

El autor declara no tener ningún conflicto de interés en la realización de este trabajo.

\section{Financiamiento}

El autor declara no haber recibido financiamiento ni apoyo alguno para la realización de este artículo.

\section{Responsabilidades éticas}

El autor declara que no existe alguna responsabilidad ética, pues no fue necesario manejar información confidencial de humanos y tampoco manejo no ético de animales de experimentación.

\section{Bibliografía}

1. Cortina A. Ética sin moral. Novena edición. España: Tecnos; 2010.

2. Bartra R. Cerebro y libertad. Ensayo sobre la moral, el juego y el determinismo. Primera reimpresión. México: Fondo Cultura Económica; 2013.

3. Bartra R. Antropología del cerebro. La ciencia y los sistemas simbólicos. Tercera reimpresión. México: Fondo Cultura Económica; 2012.

4. García-Vigil JL, García-Mangas JA Ocampo-Martínez J, Martínez-González A. Declaración de principios éticos del educador en medicina. Rev Med Inst Mex Seguro Soc. 2011;49:571-574.

5. Isola NJ. Humanizar la inteligencia artificial. Revista Expansión [Internet]. 2020 Sep 11.

6. Clark C. Los experimentos revelan por qué los robots similares a los humanos provocan sentimientos extraños. Rev Tech Explore. 2020

7. Taylor C. Algorithms control your online life. Here's how to reduce their influence [Internet]. Mashable. 2020.

8. DW.com [Internet]. Alemania: Educado pero amoral: GPT-3, inteligencia artificial capaz de escribir libros; 2020 Sep 08

9. Sigal S. You can buy a robot to keep your lonely grandparents company. Should you? Vox [Internet]. 2020 Dic 08.

10. Sturges F. How Netflix film The Social Dilemma probes the dark side of tech. Financial Times [Internet]. 2020 Sep 07.

11. López-de Mántaras R. Ética en la inteligencia artificial. Invest Cienc. 2017;491:11.

12. Instituto de Investigación en Inteligencia Artificial [Internet]. España: Declaración de Barcelona para el Adecuado Desarrollo y Uso de la Inteligencia artificial en Europa; 2017.

13. Yuval NH. Homo Deus. México: Penguin Random House LLC; 2018. 